\title{
Approach to the 4/5 law in homogeneous isotropic turbulence
}

\author{
By R. A. ANTONIA AND P. BURATTINI \\ Discipline of Mechanical Engineering, University of Newcastle, NSW 2308, Australia
}

(Received 29 August 2005 and in revised form 6 December 2005)

Kolmogorov's similarity hypotheses and his $4 / 5$ law are valid at very large Reynolds numbers. For flows encountered in the laboratory, the effect of a finite Reynolds number and of the non-stationarity or inhomogeneity associated with the large scales can affect the behaviour of the scales in the inertial range significantly. This paper focuses on the source of inhomogeneity in two types of flows, those dominated mainly by a decay of energy in the streamwise direction and those which are forced, through a continuous injection of energy at large scales. Results based on a parameterization of the second-order velocity structure function indicate that the normalized third-order structure function approaches $4 / 5$ much more rapidly for forced than for decaying turbulence. This trend is supported by grid turbulence measurements and numerical data in a periodic box.

\section{Introduction}

The similarity hypotheses proposed by Kolmogorov (1941b) (or K41) and their subsequent revision (Kolmogorov 1962, or K62) to account for intermittency have had a major impact on turbulence research. A fundamental element of these hypotheses is the assumption that the small-scale motion, which includes dissipative and inertialrange scales, is isotropic. Also, K41 and K62 require that the Reynolds number is very large so that the small-scale motion is independent of the invariably anisotropic largescale motion. The major outcome of the first similarity hypothesis is the prediction

$$
\left\langle\left(\delta u^{*}\right)^{n}\right\rangle=f_{u n}\left(r^{*}\right),
$$

where the increment $\delta u \equiv u(x+r)-u(x)$ ( $u$ is the velocity fluctuation along $x$; the separation $r$ is aligned with $x$, and the angular brackets denote averaging). For each value of $n, f_{u n}$ is a universal function, in the sense that it is expected to depend only on $r^{*} \equiv r / \eta\left(\eta \equiv\left(v^{3} /\langle\epsilon\rangle\right)^{1 / 4}\right.$ is the Kolmogorov length scale, $v$ is the kinematic viscosity and $\langle\epsilon\rangle$ is the mean energy dissipation rate). The asterisk denotes normalization by the Kolmogorov velocity scale $u_{K}\left(\equiv v^{1 / 4}\langle\epsilon\rangle^{1 / 4}\right)$ and/or $\eta$. The second similarity hypothesis yields the famous inertial-range ( $\eta \ll r \ll L ; L$ is the integral length scale) result

$$
\left\langle\left(\delta u^{*}\right)^{n}\right\rangle=C_{u n} r^{* n / 3},
$$

when $\mathrm{K} 41$ is used ( $C_{u n}$ is a universal constant).

An important exact relation between $B_{u u} \equiv\left\langle(\delta u)^{2}\right\rangle$ and $B_{u u u} \equiv-\left\langle(\delta u)^{3}\right\rangle$ was obtained by Kolmogorov (1941a), starting with the Kármán-Howarth (von Kármán \& Howarth 1938) equation for homogeneous isotropic turbulence,

$$
B_{u u u}=\frac{4}{5}\langle\epsilon\rangle r-6 v \frac{\partial}{\partial r} B_{u u} .
$$


In the inertial range, the viscous term can be neglected and (1.3) reduces to the $4 / 5$ law,

or $B_{u u u}^{*}=4 r^{*} / 5$.

$$
B_{u u u}=\frac{4}{5}\langle\epsilon\rangle r
$$

It is important to underline that (1.3) and (1.4), as well as the hypotheses in K41 and $\mathrm{K} 62$, apply only at very large Reynolds numbers. It is not surprising, therefore, that for flows normally encountered in the laboratory, (1.1) appears to be satisfied only in the dissipative range (typically $r^{*}<10$ ) (see $\$ 7$ of Chassaing et al. 2002), although the evidence is not altogether convincing especially when the isotropic form of $\langle\epsilon\rangle$,

$$
\langle\epsilon\rangle_{\text {iso }}=15 v\left\langle(\partial u / \partial x)^{2}\right\rangle,
$$

is used in forming $\delta u^{*}$ and $r^{*}$. With a few exceptions, the laboratory data also indicate an asymptotic approach to (1.2) and (1.4) as the Reynolds number (usually represented by $R_{\lambda}$ and defined by $\left\langle u^{2}\right\rangle^{1 / 2} \lambda / v$, where $\lambda$ is the Taylor microscale $\left\langle u^{2}\right\rangle^{1 / 2} /\left\langle(\partial u / \partial x)^{2}\right\rangle$ ) increases. When $R_{\lambda}$ is finite, deviations from (1.2)-(1.4) and indeed (1.5) can be quite significant. For a fixed $R_{\lambda}$, the deviations may also depend on the nature of the flow, thus casting doubt on any claim of universality, at least for scales extending beyond the dissipative range. For the same flow and $R_{\lambda}$, departures from (1.2) and (1.5) can still depend on the initial conditions that are used. It seems reasonable to ascribe these deviations to a lack of homogeneity in laboratory flows, the source of inhomogeneity depending on a number of parameters, such as the Reynolds number, type of flow and initial conditions. For the relatively large Reynolds numbers which occur in the atmospheric surface layer, the evidence in support of the ' $4 / 5$ ' law is rather inconclusive. This is partly due to the uncertainty in estimating $\langle\epsilon\rangle$. The data of Sreenivasan \& Dhruva (1998) for $R_{\lambda} \simeq 10^{4}$ indicated, however, that there is no discernible range over which $\partial B_{u u u} / \partial r$ is constant over a convincing range. This is cause for concern since the existence of the inertial range has been traditionally linked to the linear increase of $B_{\text {uuu }}$ with $r$. These authors further noted that an inertial range could not be identified unambiguously from the local slopes of the even-order moments of $\delta u$. They also stressed that the scaling of $\left\langle(\delta u)^{n}\right\rangle$ cannot be assessed effectively without first understanding the effects of finite shear and finite $R_{\lambda}$. These observations fuel speculation about the validity of the scaling exponents that have been inferred from laboratory data and also the corresponding inferences regarding the departures of these exponents from the K41 or K62 predictions.

In deriving (1.3), Kolmogorov ignored the non-stationarity term in the KármánHowarth equation. If $\partial / \partial t$ is of order $\langle\epsilon\rangle /\left\langle u^{2}\right\rangle$, then it can be readily shown that $\partial\left\langle(\delta u)^{2}\right\rangle / \partial t$ is negligible, provided $\left\langle\left(\delta u^{*}\right)^{2}\right\rangle / R_{\lambda} \ll 1$ or $\left\langle(\delta u)^{2}\right\rangle /\left\langle u^{2}\right\rangle \ll 1$ (Saffman 1968). The first of these requirements is satisfied when $R_{\lambda}$ is very large. The second can be satisfied even at relatively small $R_{\lambda}$, provided $r$ is small compared to $L$. This latter requirement is of interest since it provides some insight into why Kolmogorov's first similarity hypothesis continues to be relevant at least when $r$ lies in the dissipative range. It also reinforces the possibility that this range may have some claim to universality. The non-stationarity has since been considered by a number of authors (e.g. Danaila et al. 1999; Lindborg 1999; Lundgren 2003) in the context of decaying grid turbulence (which, strictly, is only locally homogeneous because the spatial decay precludes global homogeneity). There have also been attempts at identifying this nonstationarity in more complicated flows, e.g. the centreline region of a fully developed channel flow (Danaila et al. 2001), a homogeneous uniform shear flow (Casciola et al. 2003; Danaila, Antonia \& Burattini 2004; Qian 1999, 2002) and the region near the 
axis of a circular jet (Danaila et al. 2004). Stationary isotropic turbulence is often studied numerically by adding a forcing term to the Navier-Stokes equation for low wavenumbers (e.g. Gotoh, Fukayama \& Nakano 2002). Forcing has also been used in experiments (e.g. the flow in a cylindrical container between counter-rotating end disks, Moisy, Tabeling \& Willaime 1999) in order to achieve stationarity.

In this paper, the focus is mainly on the importance of the non-stationarity (or inhomogeneity) which is neglected in (1.3). Specifically, we distinguish between flows characterized by a decay of energy and those which are forced and examine in some detail how the $4 / 5$ law may be reached. In grid turbulence, the turbulent energy at each scale decays in the streamwise direction. Temporally decaying as well as stationary turbulence have been numerically simulated within a periodic box. Although Qian (1999) and Lundgren (2003) have considered analytically the approach to the $4 / 5$ law for both decaying and forced turbulence, they did not compare their predictions with experimental or numerical results. A comparison is provided in this paper using available data as well as new data in grid turbulence; for the latter data, the nonstationarity is determined as accurately as possible, whilst measurements at relatively small $R_{\lambda}$ of $B_{u u u} /\langle\epsilon\rangle r$ are compared with analytical results based on a more realistic model of $B_{u u}$ than considered by Lundgren (2003).

\section{Experimental and numerical data - comparison with model}

When decay or forcing is taken into account, the transport equation for $B_{u u}$ is

$$
B_{\text {иии }}=\frac{4}{5}\langle\epsilon\rangle r-6 v \frac{\partial B_{u u}}{\partial r}-I_{u}
$$

where $I_{u}$ reflects the influence of the non-stationarity. Like the Kármán-Howarth equation, (2.1) expresses the budget of the energy for any particular scale. In spatially decaying grid turbulence, $I_{u}$ can be written as (Danaila et al. 1999)

$$
I_{u}=-3 \frac{U}{r^{4}} \int_{0}^{r} s^{4} \frac{\partial B_{u u}}{\partial x} \mathrm{~d} s,
$$

where $U$ is the mean velocity in the streamwise direction $x$ and $s$ is a dummy integration variable. Since $I_{u}$ is positive, its appearance in (2.1) maintains the magnitude of $B_{\text {иии }}$ below its asymptotic value of $4\langle\epsilon\rangle r / 5$. As a consequence, measured or calculated values of $B_{u u u}^{*} / r^{*}$ cannot exceed $4 / 5$, unless $\langle\epsilon\rangle$ is evaluated incorrectly. $I_{u}$ can be estimated directly when the temporal or spatial decay of $B_{u u}$ is known. Such information is readily available from DNS data and can be acquired for grid turbulence when measurements of $B_{u u}$ are made at sufficiently fine steps in the $x$ direction. When such measurements are not available, the assumption of similarity allows $\partial B_{u u} / \partial x$ to be approximated satisfactorily. As noted earlier, the non-stationarity can be neglected either when $r \ll L$ or when $R_{\lambda} \rightarrow \infty$. In either case, it can be readily shown (Batchelor 1947) that self-similarity of $B_{u u}$ and $B_{u u u}$ based on the Kolmogorov variables $u_{K}$ and $\eta$ satisfies (1.3). This result provides strong justification for the first similarity hypothesis of Kolmogorov (1941b). When the non-stationarity is retained, George (1992) postulated an equilibrium similarity, or similarity for all scales in homogeneous isotropic turbulence, based on the Taylor microscale $\lambda$ and the energy $\left\langle u^{2}\right\rangle$. When $R_{\lambda} \rightarrow \infty$, this postulate is fully consistent with Kolmogorov similarity. It is however less accurate than Kolmogorov similarity when $R_{\lambda}$ is finite and $r$ is small, as was demonstrated by Antonia \& Orlandi (2004), since the effect of $I_{u}$ is negligible under these conditions. As the effect of $I_{u}$ increases, with increasing $r$, similarity based 

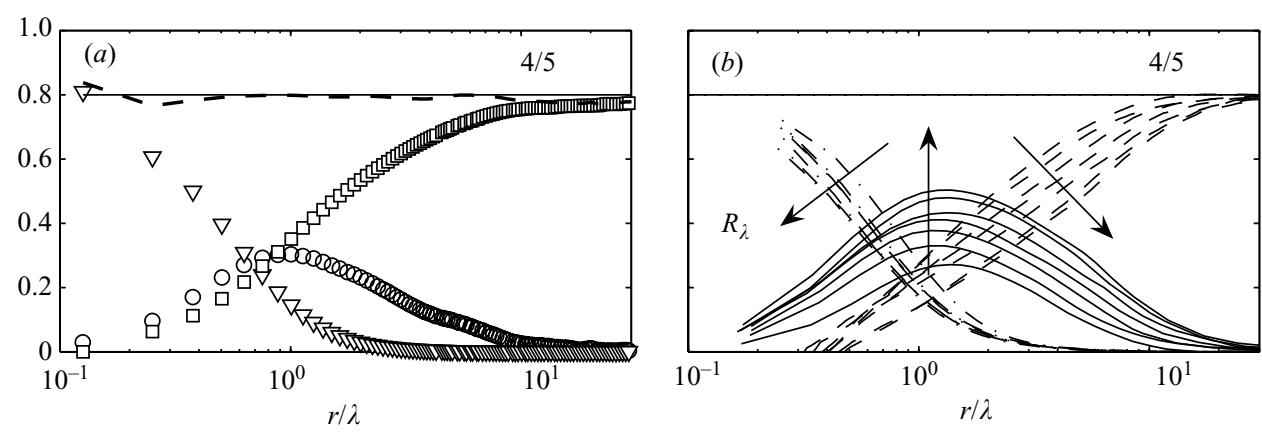

FIGURE 1. (a) Terms in (2.1), divided by $\langle\epsilon\rangle r$, for grid turbulence $\left(R_{\lambda} \simeq 40\right)$. $\nabla$, viscous term; $\bigcirc$, third-order structure function; $\square, I_{u}$; solid horizontal line: $4 / 5$; dashed line: sum of the viscous term, third-order structure function term and $I_{u} .(b)$ Variation with $R_{\lambda}$ of the terms in (2.1), divided by $\langle\epsilon\rangle r$, for the grid turbulence data of Zhou \& Antonia (2000) $\left(R_{\lambda}\right.$ increases between 27 and 100 in the direction of the arrows). Solid lines: $B_{\text {uиu }} /\langle\epsilon\rangle r$; dashed lines: $I_{u}$ (calculated by difference after assuming the validity of (2.1)); dash-dotted lines: viscous term; solid horizontal line: $4 / 5$.

on $\lambda$ and $\left\langle u^{2}\right\rangle$ becomes more relevant. For this reason, we have chosen to estimate $I_{u}$ with the use of George's similarity proposal. In grid turbulence, the expression for $I_{u}$ is

$$
I_{u}=\frac{3 U}{r^{4}}\left[\lambda^{5} \frac{\mathrm{d}\left\langle u^{2}\right\rangle}{\mathrm{d} x} \int_{0}^{r / \lambda}\left(\frac{s}{\lambda}\right)^{4} \frac{B_{u u}}{\left\langle u^{2}\right\rangle} \mathrm{d}\left(\frac{s}{\lambda}\right)-\left\langle u^{2}\right\rangle \frac{\mathrm{d} \lambda}{\mathrm{d} x} \lambda^{4} \int_{0}^{r / \lambda}\left(\frac{s}{\lambda}\right)^{5} \frac{\partial}{\partial(r / \lambda)} \frac{B_{u u}}{\left\langle u^{2}\right\rangle} \mathrm{d}\left(\frac{s}{\lambda}\right)\right] .
$$

Its detailed derivation can be found in Antonia et al. (2003). The terms in (2.1) have been measured in the turbulence generated by a biplane grid composed of square rods (solidity 0.35$)$ using an X-wire located at $x / M=52\left(R_{\lambda} \simeq 40\right)$. For this flow, $\langle\epsilon\rangle$ was obtained reliably from the turbulent energy budget

$$
\langle\epsilon\rangle=-\frac{1}{2} U \frac{\mathrm{d}\left\langle q^{2}\right\rangle}{\mathrm{d} x},
$$

where $\left\langle q^{2}\right\rangle=\left\langle u^{2}\right\rangle+\left\langle v^{2}\right\rangle+\left\langle w^{2}\right\rangle$. Here, $\left\langle q^{2}\right\rangle$ was approximated by $\left\langle q^{2}\right\rangle=\left\langle u^{2}\right\rangle+2\left\langle v^{2}\right\rangle$ since $\left\langle v^{2}\right\rangle \simeq\left\langle w^{2}\right\rangle$. The results are shown in figure 1(a) after normalizing by $\langle\epsilon\rangle$.

The imbalance, or difference between $4 / 5$ and the sum of the remaining normalized terms in (2.1), is negligible at nearly all values of $r / \lambda$, reflecting partly the appropriateness of the similarity assumption used to estimate $I_{u}$ and also the accuracy with which $\langle\epsilon\rangle$ has been obtained. As noted by Antonia et al. (2000b), the presence of $I_{u}$ in (2.1) allows compliance with two important results. The energy budget, (2.4), is retrieved at large $r$, whilst the decay of $\langle\epsilon\rangle$ or, equivalently, the decay of enstrophy in homogeneous turbulence, is correctly reproduced in the limit of $r \rightarrow 0$. At $r \approx \lambda$, (2.1) is almost perfectly satisfied by the data, implying that $\langle\epsilon\rangle$ can be estimated from this equation only from a knowledge of $B_{u u}$ and $B_{u u u}$ for values of $r$ close to $\lambda$. That the influence of $I_{u}$ on scales corresponding to the maximum value of the normalized third-order structure function, i.e.

$$
A_{u}=\max \left(B_{u u u} /\langle\epsilon\rangle r\right),
$$

should diminish as $R_{\lambda}$ increases can be readily inferred from figure $1(b)$; here the data in Zhou \& Antonia (2000) have been used. Although the range of $R_{\lambda}$ is limited, the trend is unmistakable. The implication is that the onset of a scaling range becomes 


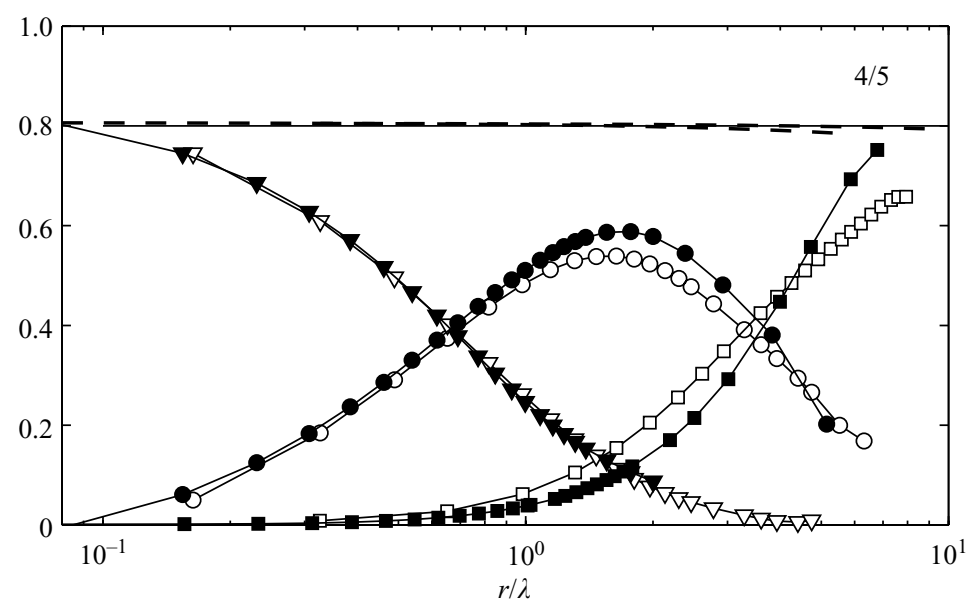

Figure 2. Terms in (2.1), divided by $\langle\epsilon\rangle r$, using the DNS data of Fukayama et al. (2000) at roughly the same $R_{\lambda}(\simeq 70)$. Solid symbols: forced turbulence; open symbols: temporally decaying turbulence. $\bigcirc$, Third-order structure function; $\square, I_{u} ; \nabla$, viscous term; dashed lines: sum of the viscous term, third-order structure function term and $I_{u}$; solid horizontal line: $4 / 5$.

more realistic with increasing $R_{\lambda}$. Near $r=\lambda$, the magnitude of $I_{u}$, estimated here from the measured values of $B_{u u}^{*}$ and $B_{u u u}^{*}$ and by assuming the validity of (2.1), decreases with $R_{\lambda}$ at least as rapidly as that of the viscous term.

For a fixed $R_{\lambda}, I_{u} /\langle\epsilon\rangle r$ is unlikely to be universal, even for a given type of flow. In grid turbulence, $I_{u} /\langle\epsilon\rangle r$ has been found to depend, but only slightly, on the shape and solidity of the grid (Lavoie et al. 2005). Antonia \& Burattini (2004) pointed out that for the wake data of Antonia, Zhou \& Romano (2002), obtained at the same distance $(x / D=70)$ downstream of 5 different wake generators, the magnitude and shape of $B_{\text {uuu }} /\langle\epsilon\rangle r$ can vary significantly, even though $R_{\lambda}$ was nominally the same $(\simeq 200)$ in each case. The implication is that $I_{u} /\langle\epsilon\rangle r$ can vary between differently generated wakes, reflecting the different degrees of organization in each wake.

For forced turbulence, Moisy et al. (1999) assumed (following Novikov 1993)

$$
I_{u}=\frac{2}{7}\langle\epsilon\rangle \frac{r^{3}}{L_{f}^{2}},
$$

where $L_{f}$ is an integral scale which characterizes the forcing and was found to be nearly constant. The value of $\langle\epsilon\rangle$ was obtained via (2.1) and (2.6) by fitting to the measured third-order structure function. Increasing $L_{f}$ corresponds to stirring the flow at relatively larger scales. $L_{f}$ is limited by the size of the test section in experiments and the size of the DNS box. Gotoh et al. (2002) and Fukayama et al. (2000) used

$$
I_{u}=\frac{2}{35} r^{3} \int_{0}^{\infty} k^{2} F(k) \mathrm{d} k
$$

in the context of DNS for forced box turbulence. Here, $F(k)$ is the spectrum of the random force, constant over a small, low-wavenumber range and zero elsewhere. Fukayama et al. (2000) presented all the terms of (2.1) for $R_{\lambda} \simeq 70$. Their data are plotted in figure 2 using a linear scale for the ordinate and $r / \lambda$ along the abscissa. The isotropic relation $\lambda / \eta=15^{1 / 4} R_{\lambda}^{1 / 2}$ was used to convert the values of $r / \eta$ given by Fukayama et al. (2000). They also carried out simulations of temporally decaying box turbulence; their results for $R_{\lambda} \simeq 70$ are included in figure 2 . Although there is practically no difference between the viscous terms, the shape of $I_{u}$ differs between 

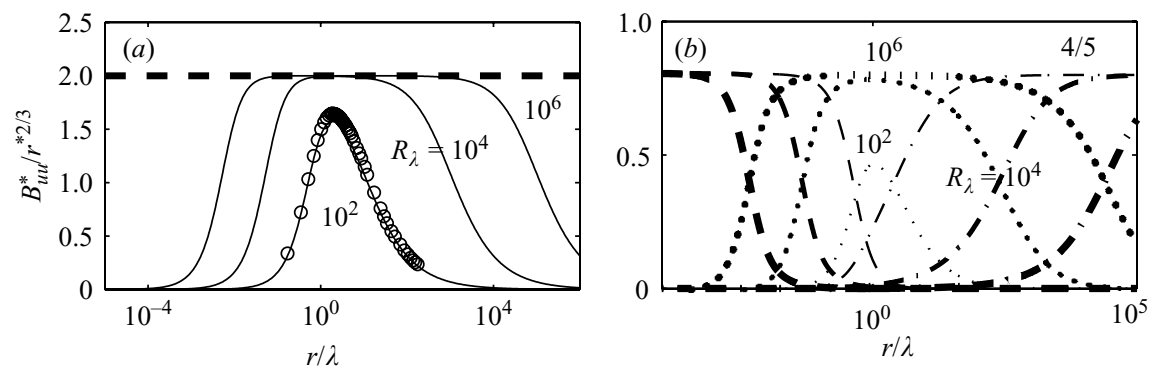

FIGURE 3. (a) Compensated second-order structure function. Solid lines: model (2.8); O, measured grid turbulence data of Zhou \& Antonia (2000) at $R_{\lambda}=100$; dashed line: asymptotic value corresponding to $C_{u 2}$ and model of Lindborg (1999) and Lundgren (2003). (b) Variation with $R_{\lambda}$ of the terms in (2.1), divided by $\langle\epsilon\rangle r$, in decaying isotropic turbulence assuming (2.8). Dotted line: third-order structure function; dash-dotted line: $I_{u}$; dashed line: viscous term.

the two cases. With forcing, $I_{u}$ is smaller for small separations and rises steeply at large separations. As a consequence, $A_{u}$ is bigger and located at slightly larger $r / \lambda$. In decaying turbulence, $B_{u u}$ approaches a plateau slowly, at large $r$. In forced turbulence, the shape of $B_{u u}$ varies according to the type of forcing that is applied. To examine the dependence on $R_{\lambda}$ of all terms in (2.1), we follow the approach of Antonia et al. (2003) who used a description of $B_{u u}^{*}$ (Kurien \& Sreenivasan 2000) which extends from the smallest (Kolmogorov) length scale to the integral length scale $L$,

$$
B_{u u}^{*}=\frac{r^{* 2}}{15} \frac{\left(1+\beta r^{*}\right)}{\left(1+\left(r^{*} / r_{c u}^{*}\right)^{2}\right)^{c}} .
$$

Here, $r_{c u}^{*}$ is identified with the crossover between the dissipative and inertial ranges, $c \equiv\left(1-\zeta_{u} / 2\right)$ and $\beta \equiv L^{*-1}$. Equation (2.8) is a modification, for finite Reynolds numbers, of the model for $B_{u u}^{*}$ first proposed by Batchelor (1951) with $\beta=0$, figure 3 . For isotropic turbulence, $\lambda^{*}=15^{1 / 4} R_{\lambda}^{1 / 2},\left\langle u^{* 2}\right\rangle=R_{\lambda} / 15^{1 / 2}$ and $L^{*} \equiv 15^{-3 / 4} C_{\epsilon} R_{\lambda}^{3 / 2}$, where $L$ has been identified with $C_{\epsilon}\left\langle u^{2}\right\rangle^{3 / 2} /\langle\epsilon\rangle$. The dimensionless energy dissipation rate parameter $C_{\epsilon}$ is expected to become constant at sufficiently large $R_{\lambda}$ but its magnitude should depend on the initial conditions. In general, one expects the shape of $B_{u u}^{*}$ to depend on the type and level of organization in a particular flow (e.g. Lavoie et al. 2005), which most likely reflects the influence of the initial conditions. A value of 1 for $C_{\epsilon}$ was assumed, as in Antonia et al. (2003). We have also assumed that $r_{c u}^{*}=\left(15 C_{u 2}\right)^{3 / 4}$, with a value of 2 for the Kolmogorov constant $C_{u 2}$ while the K41 value of $2 / 3$ was assigned to $\zeta_{u}$. Although $C_{u 2}, \zeta_{u}$ and $r_{c u}^{*}$ may vary slightly with $R_{\lambda}$ (e.g. Antonia, Pearson $\&$ Zhou $2000 a$ ) when $R_{\lambda}$ is small, the present estimates of $I_{u}$ and $B_{u u u}$ should be sufficiently accurate to provide a reasonable indication of how Kolmogorov's 4/5 law is approached. Figure 3(a) shows that $B_{u u}^{*} / r^{* 2 / 3}$ becomes close to 2 (the Kolmogorov constant) at $R_{\lambda} \simeq 10^{4}$, although a reasonably sized plateau for this quantity is achieved only at $R_{\lambda} \simeq 10^{6}$. Lindborg (1999) and Lundgren (2003) assumed, using Kolmogorov's second similarity hypothesis, that

$$
B_{u u}=C_{u 2}\left\langle u^{2}\right\rangle\left(\frac{r}{L}\right)^{2 / 3}=C_{u 2}(\langle\epsilon\rangle r)^{2 / 3}
$$

with $C_{u 2}=2$. This approach neglects both the dissipative range and the $R_{\lambda}$-dependence of the inertial range. The grid turbulence data $\left(R_{\lambda}=100\right)$ of Zhou \& Antonia (2000) have been included in figure $3(a)$ to show that (2.8) approximates the measured data adequately. 


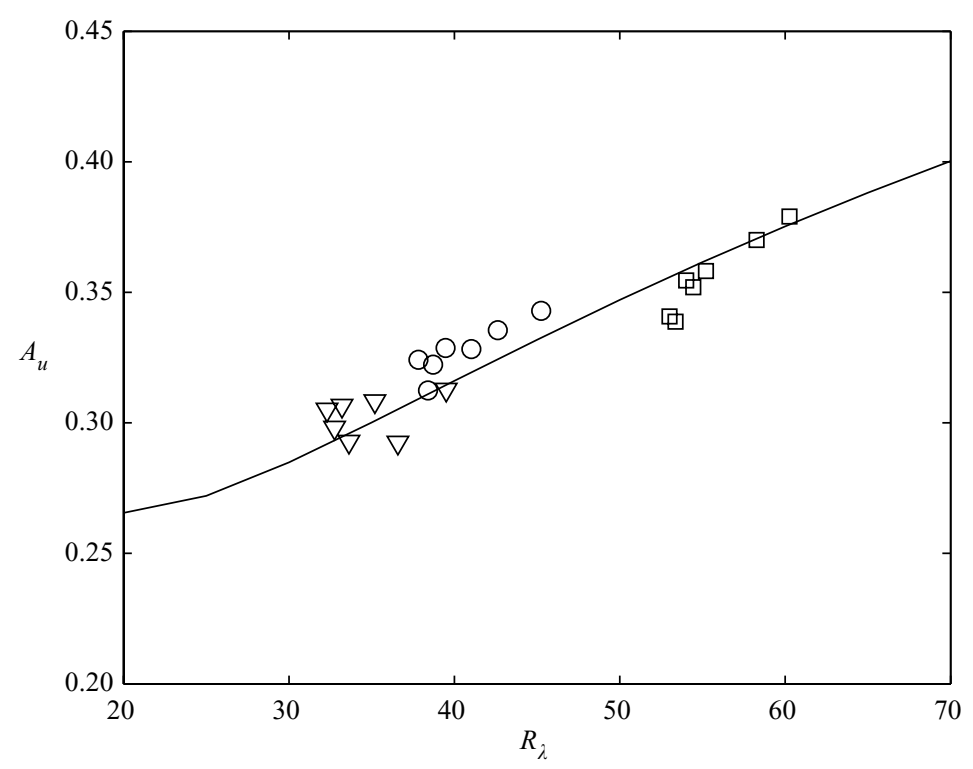

FiguRE 4. Variation of $A_{u}$ with $R_{\lambda}$ for $R_{\lambda} \lesssim 60$. Solid line: based on (2.8), see text for details; symbols, grid turbulence behind biplane grids of different shapes and solidities (Lavoie et al. 2005): $\nabla$, round bars, solidity 0.35 ; $\bigcirc$, round bars, solidity $0.44 ; \square$ : square bars, solidity 0.35 .

$B_{u u u}$ was evaluated after introducing (2.8) into (2.1), and assuming a value of the decay rate exponent $m$ for the turbulent kinetic energy of -1.25 (close to that measured by Antonia et al. 2003). This is shown in figure 3(b), where the viscous term and $I_{u}$ are also included. $\dagger$ As $R_{\lambda}$ increases, the latter two shift to the left and right respectively, and $B_{\text {uиu }}$ appears to exhibit a convincing plateau only when $R_{\lambda}$ is about $10^{6}$. Experimental values of $A_{u}$ in low- $R_{\lambda}(\lesssim 60)$ grid turbulence are compared with the model in figure 4 . The data were obtained for different grid geometries and solidities (see Lavoie et al. 2005). Notwithstanding the scatter in the data for each of the grids, the small effect of the different initial conditions at the grids, and the lack of information in the model on the effect of initial conditions, the agreement between the model and the data is satisfactory, lending support to (2.8) and the hypotheses assumed in applying (2.2) to grid turbulence. The agreement also reflects the fact that $\langle\epsilon\rangle$ was estimated accurately via (2.4) for these data, and measurements were made at small intervals (equal to $M$ ) in the $x$-direction. This approach eliminated the need to use a similarity hypothesis since $\partial B_{u u} / \partial x$ in (2.2) could be estimated directly. For all data in figure 4 , the departure from isotropy, as measured by the ratio $\left\langle u^{2}\right\rangle /\left\langle v^{2}\right\rangle$ say, is of order $10 \%$; using a secondary contraction downstream of each grid resulted in this ratio being closer to 1 but the magnitude of $A_{u}$ was essentially unchanged (Lavoie 2005). The solid curve in figure 4 has been extended to much higher values of $R_{\lambda}$ in figure 5. Included in figure 5 are box turbulence data as well as previously published grid turbulence data (for clarity, the data in figure 4 are not shown). Clearly, $4 / 5$ is reached much more rapidly for the forced than for the decaying data, the values of $R_{\lambda}$ that are required being about $10^{3}$ and $10^{6}$ respectively. As already noted from the distributions in figure 2 at the same $R_{\lambda}$, the difference in the trends can be attributed to zero at small and large $r$ (figures 1 and 2 of Lindborg (1999) and figure 1 of Lundgren (2003)). 


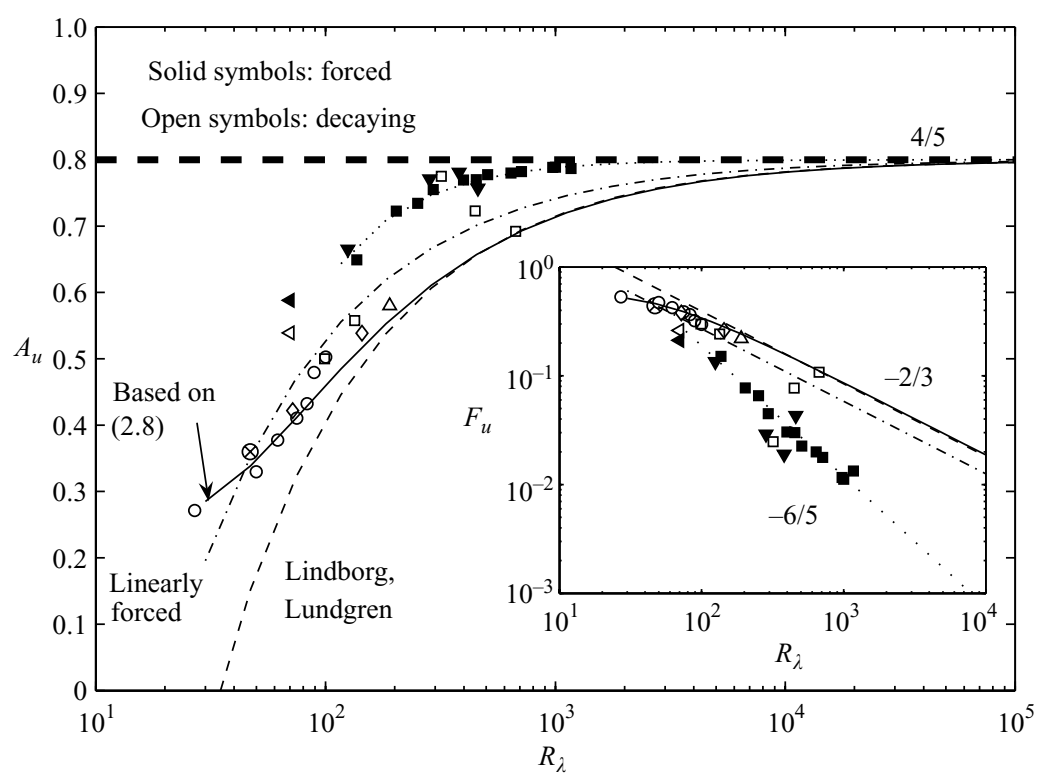

FIGURE 5. Variation with $R_{\lambda}$ of $A_{u}$. Solid line: based on (2.8); $\boldsymbol{\nabla}$, DNS data for forced box turbulence (Gotoh et al. 2002); 4, DNS data for forced box turbulence (Fukayama et al. 2000); $\triangleleft$, DNS data for decaying box turbulence (Fukayama et al. 2000); $\otimes$, DNS data for decaying box turbulence (Orlandi \& Antonia 2002); $\mathbf{\square}$, measurements of Moisy et al. (1999) for forced turbulence; $\diamond$, grid turbulence (Gagne et al. 2004); , grid turbulence (Zhou \& Antonia 2000); $\triangle$, grid turbulence (van de Water \& Herweijer 1999); $\square$, active grid turbulence (Mydlarski \& Warhaft 1996); solid line: model based on (2.8); dash-dotted line: model of Lundgren (2003) for linearly forced turbulence; dashed line: model of Lindborg (1999) and Lundgren (2003) for decaying turbulence; dotted line: fit to the data of Moisy et al. (1999); dashed horizontal line: 4/5. Inset: $F_{u} \equiv\left(4 / 5-A_{u}\right)$ as a function of $R_{\lambda}$ on a log-log scale. The slope of the dashed and dash-dotted lines is -2/3 (Lindborg 1999; Lundgren 2003; Qian 1999), while the slope of the dotted line is -6/5 (Moisy et al. 1999; Qian 1999).

to $I_{u}$ (although the difference between the two values of $A_{u}$ in figure 2 is small, it is nonetheless significant, as noted by the authors). The agreement with the DNS data of Orlandi \& Antonia (2002) and the grid turbulence data of Gagne et al. (2004), Zhou \& Antonia (2000) and van de Water \& Herweijer (1999) is good (for the latter, only the data at the centre of the working section were used). However, there is poor agreement with some of the active grid turbulence data of Mydlarski \& Warhaft (1996), which exhibit a large scatter, reflecting the uncertainty in estimating $\langle\epsilon\rangle$ in those experiments. For $R_{\lambda} \gtrsim 200$, the model becomes almost indistinguishable from the predictions of Lindborg (1999) and Lundgren (2003). For smaller values of $R_{\lambda}$, the present model (based on (2.8)) is superior because it accounts for the restricted scaling range, vis-à-vis (2.9). In (2.9), the assumed values of $B_{u u}^{*}$ are consistently higher than those of (2.8) (see figure $3 a$ ). For $R_{\lambda} \gtrsim 40$, results for linearly forced turbulence (Lundgren 2003) lie between those for decaying and forced turbulence; this is not surprising given that the energy level is sustained across all turbulent scales. The dotted line was provided by Moisy et al. (1999) as a fit to their data. The inset in figure 5 shows the variation of $F_{u} \equiv\left(4 / 5-A_{u}\right)$ on a $\log -\log$ scale, as used by Zhou et al. (2000). For $R_{\lambda} \gtrsim 100$, the data follow two separate power-law regimes. For decaying turbulence, the power-law exponent is $-2 / 3$ as predicted by Lindborg (1999), Lundgren (2003) and Qian (1999). The latter author arrived at this result by 
using the spectral equation and a model for the turbulent energy spectrum which has a slope of $-5 / 3$ in the inertial range. The present model is also consistent with the $-2 / 3$ power-law behaviour when $R_{\lambda}$ exceeds about 200; below this value, it indicates a much slower rate of decay. Qian (1999) emulated forcing by considering a Dirac $\delta$-function injection of energy at small wavenumbers and predicted a decay exponent for $F_{u}$ of $-6 / 5$, in accord with the data of Gotoh et al. (2002) and Moisy et al. (1999), although it should be kept in mind that the latter authors did not measure $\langle\epsilon\rangle$ directly in (2.1).

\section{Concluding comments}

The results in figure 5 indicate that the asymptotic value of $4 / 5$ is approached more rapidly for forced than for decaying turbulence. Correspondingly, the Kolmogorov inertial range is likely to be established more rapidly when forcing is applied; this vindicates the use of forcing for emulating Kolmogorovian turbulence. Figures 3 and 5 indicate that, for a substantial range to exist, $R_{\lambda}$ should exceed about $10^{3}$ when forcing is applied and $10^{6}$ when the turbulence is decaying; the latter estimate is consistent with that proposed by Antonia et al. (2003). The difference in the magnitude of $A_{u}$, the maximum value of $B_{u u u} /\langle\epsilon\rangle r$, between the two cases reflects a difference in the magnitude of $I_{u}$, the non-stationarity or inhomogeneity in (2.1). This difference suggests that turbulence statistics associated with scales for which $B_{u u u} /\langle\epsilon\rangle r$ is close to its maximum are unlikely to be universal, at least for Reynolds numbers normally encountered in the laboratory. Consequently, results for forced and decaying turbulence should be interpreted in the context of this difference, even when they have been obtained at the same $R_{\lambda}$. It is worth pointing out that the present difference between forced and decaying results was anticipated by Lindborg (1999) who suggested that experiments aimed at testing intermittency should be carried out in stationary flows.

The support of the Australian Research Council is acknowledged. We are especially grateful to L. Danaila for her contribution to various aspects of this work.

\section{REFERENCES}

Antonia, R. A. \& Burattini, P. 2004 Small-scale turbulence: How universal is it? In 15th Australasian Fluid Mechanics Conference (ed. M. Behnia, W. Lin \& G. D. McBain), p. AFMC00184. The University of Sydney.

Antonia, R. A. \& OrLandi, P. 2004 Similarity of decaying isotropic turbulence with a passive scalar. J. Fluid Mech. 505, 123-151.

Antonia, R. A., Pearson, B. R. \& Zhou, T. $2000 a$ Reynolds number dependence of second-order velocity structure functions. Phys. Fluids 12, 3000-3006.

Antonia, R. A., Smalley, R. J., Zhou, T., Anselmet, F. \& Danaila, L. 2003 Similarity of energy structure functions in decaying homogeneous isotropic turbulence. J. Fluid Mech. 487, 245269.

Antonia, R. A., Zhou, T., Danaila, L. \& Anselmet, F. $2000 b$ Streamwise inhomogeneity of decaying grid turbulence. Phys. Fluids 12, 3086-3089.

Antonia, R. A., Zhou, T. \& Romano, G. P. 2002 Small-scale turbulence characteristics of twodimensional bluff body wakes. J. Fluid Mech. 459, 67-92.

Batchelor, G. K. 1947 Kolmogoroff's theory of locally isotropic turbulence. Proc. Camb. Phil. Soc. 43, 533-559.

Batchelor, G. K. 1951 Pressure fluctuations in isotropic turbulence. Proc. Camb. Phil. Soc. 47, 359-374.

Casciola, C. M., Gualtieri, P., Benzi, R. \& Piva, R. 2003 Scale-by-scale budget and similarity laws for shear turbulence. J. Fluid Mech. 476, 105-114. 
Chassaing, P., Antonia, R. A., Anselmet, F., Joly, L. \& Sarkar, S. 2002 Variable Density Fluid Turbulence. Kluwer.

Danaila, L., Anselmet, F., Zhou, T. \& Antonia, R. A. 1999 A generalization of Yaglom's equation which accounts for the large-scale forcing in heated decaying turbulence. J. Fluid Mech. 391, 359-372.

Danaila, L., Anselmet, F., Zhou, T. \& Antonia, R. A. 2001 Turbulent energy scale budget equations in a fully developed channel flow. J. Fluid Mech. 430, 87-109.

Danaila, L., Antonia, R. A. \& Burattini, P. 2004 Progress in studying small-scale turbulence using 'exact' two-point equations. New J. Phys. 6 (128), 1-23.

Fukayama, D., Oyamada, T., Nakano, T., Gotoh, T. \& Yamamoto, K. 2000 Longitudinal structure functions in decaying and forced turbulence. J. Phys. Soc. Japan 69, 701-715.

Gagne, Y., Castaing, B., Baudet, C. \& Malécot, Y. 2004 Reynolds number dependence of third-order velocity structure functions. Phys. Fluids 16, 482-485.

George, W. K. 1992 The decay of homogeneous isotropic turbulence. Phys. Fluids A 4, 1492-1509.

Gotoh, T., Fukayama, D. \& Nakano, T. 2002 Velocity field statistics in homogeneous steady turbulence obtained using a high-resolution direct numerical simulation. Phys. Fluids 14, 1065-1081.

von KÁrmán, T. \& Howarth, L. 1938 On the statistical theory of isotropic turbulence. Proc. R. Soc. Lond. A 164, 192-215.

Kolmogorov, A. N. 1941 a Dissipation of energy in the locally isotropic turbulence. Dokl. Akad. Nauk SSSR 32 (1), English translation in Proc. R. Soc. Lond. A 434 (1991), 15-17.

Kolmogorov, A. N. $1941 b$ Local structure of turbulence in an incompressible fluid for very large Reynolds numbers. Dokl. Akad. Nauk SSSR 30 (4), 299-303.

Kolmogorov, A. N. 1962 A refinement of previous hypotheses concerning the local structure of turbulence in a viscous incompressible fluid at high Reynolds number. J. Fluid Mech. 13, $82-85$.

Kurien, S. \& SReEnivasan, K. R. 2000 Anisotropic scaling contributions to high-order structure functions in high-Reynolds-number turbulence. Phys. Rev. E 62, 2206-2212.

Lavoie, P. 2005 The effect of initial conditions in grid-generated turbulence. PhD thesis, University of Newcastle (in preparation).

Lavoie, P., Burattini, P., Duenidi, L. \& Antonia, R. A. 2005 Effect of initial conditions on decaying grid turbulence at low $\mathrm{R}_{\lambda}$. Exps. Fluids 39, 865-874.

Lindborg, E. 1999 Correction to the four-fifths law due to variations of the dissipation. Phys. Fluids 11, 510-512.

LundGren, T. S. 2003 Linearly forced turbulence. CTR Annual Research Briefs.

Moisy, F., Tabeling, P. \& Willaime, H. 1999 Kolmogorov equation in a fully developed turbulence experiment. Phys. Rev. Lett. 82, 3994-3997.

MydlaRSKI, L. \& WARHAFT, Z. 1996 On the onset of high-Reynolds-number grid-generated wind tunnel turbulence. J. Fluid Mech. 320, 331-368.

Novikov, E. A. 1993 Statistical balance of vorticity and a new scale for vortical structures in turbulence. Phys. Rev. Lett. 71, 2718-2720.

Orlandi, P. \& Antonia, R. A. 2002 Dependence of the non-stationary form of Yaglom's equation on the Schmidt number. J. Fluid Mech. 451, 99-108.

QIAN, J. 1999 Slow decay of the finite Reynolds number effect of turbulence. Phys. Rev. E 60, 3409-3412.

QIAN, J. 2002 Scaling of structure functions in homogeneous shear-flow turbulence. Phys. Rev. E 65, 036301.

Saffman, P. G. 1968 Lectures on homogeneous turbulence. In Topics in Nonlinear Physics (ed. N. Zabusky), pp. 485-614. Berlin: Springer.

Sreenivasan, K. R. \& Dhruva, B. 1998 Is there scaling in high-Reynolds-number turbulence? Prog. Theor. Phys. Suppl. 130, 103-120.

van De Water, W. \& HerweiJer, J. A. 1999 High-order structure functions of turbulence. J. Fluid Mech. 387, 3-37.

Zhou, T. \& Antonia, R. A. 2000 Reynolds number dependence of the small-scale structure of grid turbulence. J. Fluid Mech. 406, 81-107.

Zhou, T., Antonia, R. A., Danaila, L. \& Anselmet, F. 2000 Approach to the four-fifths 'law' for grid turbulence. J. Turb. 1 (5), 1-12. 